\title{
Reusable surface plasmon resonance biosensor chip for the detection of H1N1 influenza virus
}

\author{
Haneul Yoo ${ }^{\mathrm{a}, 1}$, Junghyun Shin ${ }^{\mathrm{a}, 1}$, Jieun Sim ${ }^{\mathrm{c}, \mathrm{d}, \mathrm{e}}$, Hyunmin Cho ${ }^{\mathrm{c}, \mathrm{e}}$, Seunghun Hong ${ }^{\mathrm{b}, *}$ \\ ${ }^{a}$ Department of Physics and Astronomy, Seoul National University, Seoul, 08826, Republic of Korea \\ b Department of Physics and Astronomy, And Institute of Applied Physics, Seoul National University, Seoul, 08826, Republic of Korea \\ c Department of Nanobiotechnology, KRIBB School of Biotechnology, University of Science and Technology, Daejeon, 34113, Republic of Korea \\ ${ }^{\mathrm{d}}$ Bionano Research Center, Korea Research Institute of Bioscience and Biotechnology (KRIBB), Daejeon, 34141, Republic of Korea \\ e BioNano Health Guard Research Center (H-GUARD), Daejeon, 34141, Republic of Korea
}

\section{A R T I C L E I N F O}

\section{Keywords}

Surface plasmon resonance

Biosensor

Reusable

Ferromagnetic pattern

Magnetic trapping-releasing

Influenza

\begin{abstract}
A B S T R A C T
We developed a reusable magnetic surface plasmon resonance (SPR) sensor chip for detecting various target molecules repeatedly in a conventional SPR system. Here, ferromagnetic patterns on a SPR sensor chip were utilized to trap a layer of magnetic particles, and they were utilized as a solid substrate for SPR sensing in a conventional SPR system. After a sensing experiment, the used magnetic particles were removed by external magnetic fields, and a new layer of magnetic particles was immobilized to the SPR sensor chip for additional sensing measurements. Since magnetic particles were trapped on the ferromagnetic patterns, we could use our reusable SPR chip for SPR sensing measurements in a traditional SPR system without any applied magnetic fields. Significantly, ferromagnetic patterns on the sensor chip surface deflected the strong external fields, so that the large aggregation of magnetic particles on the sensor surface was reduced. We demonstrated using a single reusable SPR sensor chip to measure the nucleoprotein (NP) of H1N1 influenza virus solution ranging repeatedly for more than 7 times without significant signal degradation. Also, different target molecules could be repeatedly measured in a single SPR chip. Since our reusable SPR sensor chip can be repeatedly used in a conventional SPR system without any chemical processes for refreshment, the cost for SPR sensing should be significantly reduced. In this case, our reusable SPR sensor chip can be a major breakthrough and can be used for versatile practical applications of SPR sensors.
\end{abstract}

\section{Introduction}

A surface plasmon resonance (SPR) based sensing method is a powerful tool for the real-time and label-free monitoring of biomolecular interactions(Krishnamoorthy et al., 2010; Mandenius et al., 2008; Wang et al., 2013; Wong and Olivo, 2014; Zeng et al., 2014). In SPR-based biosensors, the selective bindings of target molecules to receptors on the SPR sensor surface induced its refractive index changes, which can be measured for the label-free detection of versatile target analytes in real time(Wang et al., 2013; Wong and Olivo, 2014). Also, various SPR-based measurement systems have been used extensively for the analyses of interaction kinetics between different biomolecules such as protein-ligand, protein-protein, or DNA-protein(Jecklin et al., 2009; Karlsson and Falt, 1997; Patching, 2014; Pollet et al., 2009; Wegner et al., 2003). However, most of SPR biosensor chips are rather expensive compared with common commercial biosen- sors such as strip-type sensors. Also, it is usually very difficult to reuse a used SPR sensor chip. Such a limitation has been one of the major stumbling blocks holding back its diverse commercial applications.

Recently, several groups have developed methods for reusable SPR sensors (Choi and Chae, 2009; Hsu et al., 2015; Lee et al., 2011; Makaraviciute et al., 2015; Roche et al., 2011; Zhao et al., 2014). In one method, a used SPR sensor chip was treated chemically to remove the used receptors, and new receptor molecules were applied for addition sensing measurements(Choi and Chae, 2009; Makaraviciute et al., 2015; Zhao et al., 2014). However, such a chemical treatment often contaminated sensor surfaces and degraded the sensor performance. Previous works show that chemical treatments can damage bioreceptors, causing losses of the sensor signals (Goode et al., 2015). In another work, receptor-modified magnetic particles were assembled on a SPR sensor surface via external magnetic fields and used for SPR sensing measurements (Lee et al., 2011). After a sens-

\footnotetext{
* Corresponding author. Department of Physics and Astronomy, Seoul National University, Seoul, 08826, Republic of Korea.

E-mail address: seunghun@snu.ac.kr (S. Hong)

1 These authors contributed equally to this work.
} 
ing, magnetic fields with an opposite direction were applied to remove the magnetic particles, leaving a fresh SPR sensor surface ready for additional sensing. This method based on magnetic fields can minimize the chemical contamination. However, the strong external magnetic fields may result in the aggregation of magnetic particles and leave residues on the SPR sensor surface, which may reduce the reliability of the sensor. Furthermore, in this method, an external magnetic field should be maintained during the SPR sensing process, and, thus, a conventional SPR sensing system should be modified to include an electromagnet device for sensing measurements. Until now, it is very difficult to build a SPR-based biosensor which can be reused repeatedly in a conventional SPR systems without degrading its signals. Another powerful strategy for cost-effective SPR biosensor can be optical fiber-based SPR sensors. The use of optical fibers for SPR sensing allowed one to develop low cost and even miniaturized SPR biosensors. However, such optical fiber-based SPR biosensors usually require specifically-configured equipment and cannot be utilized in conventional SPR systems (Chiavaioli et al., 2018; Guo, 2017; Loyez et al., 2019; Shushama et al., 2017; Zhao et al., 2019).

Herein, we report a reusable magnetic SPR sensor chip which allows us to repeatedly detect target analytes such as H1N1 virus in a conventional SPR system. In this strategy, ferromagnetic patterns on a SPR chip trapped a layer of magnetic particles with a fresh surface, and they were used as a solid substrate for SPR sensing in a conventional SPR system. Then, the used magnetic particles were removed via external magnetic fields, and a new layer of fresh magnetic particles was trapped on the SPR chip for additional sensing measurements. Since the ferromagnetic patterns were holding magnetic particles, our reusable SPR chip could be used for SPR sensing measurements in a conventional SPR system without any external magnetic fields. Furthermore, the strong external magnetic fields were deflected by the ferromagnetic patterns on the sensor surface, which avoided the large aggregation of magnetic particles on the surface and enabled rather large sensing signals comparable to those of conventional SPR chips. Using a single reusable SPR sensor chip, we could repeatedly detect the nucleoprotein (NP) of H1N1 influenza virus with the protein concentration ranging from $30 \mathrm{ng} / \mathrm{ml}$ to $10 \mu \mathrm{g} / \mathrm{ml}$. Importantly, the sensing signals did not degrade even after more than 7 times of repeated SPR measurements. We also demonstrated the detection of different target molecules using a single SPR chip. Since the reusable SPR chip can be used repeatedly in a conventional SPR system and does not require any complicated chemical processes for refreshment, our method should dramatically reduce the cost for sensing processes and open up versatile practical applications of SPR sensors such as a molecular recognition study, a drug screening, a blood test, and a disease diagnosis.

\section{Materials and methods}

\subsection{Materials}

A superparamagnetic nanoparticle (nano-screenMAG-CMX) solution was purchased from Chemicell GmbH (Germany). The hydrodynamic size of a magnetic particle was about $100 \mathrm{~nm}$, and the surface of the magnetic particle was covered with carboxymethyl-dextran (CMX) having a carboxyl group. 1-Ethyl-3-(3-dimethylaminopropyl) carbodiimide hydrochloride (EDC), N-Hydroxysuccinimide (NHS), and Ethanolamine hydrochloride- $\mathrm{NaOH}$ were purchased from GE Healthcare (Sweden). SIA KIT Au containing a SPR support, double-sided adhesive tape, and a protective sheath were also purchased from GE Healthcare. BioNano Health Guard Research Center (H-GUARD) prepared nucleoprotein (NP) of influenza type A H1N1 and its corresponding monoclonal antibody. 1x PBS (pH 7.4, $154 \mathrm{mM} \mathrm{NaCl}, 5.6 \mathrm{mM} \mathrm{Na} 2 \mathrm{HPO}_{4}, 1.1 \mathrm{mM} \mathrm{KH}_{2} \mathrm{PO}_{4}$ ) was purchased from Lonza (USA).

\subsection{Fabrication of nickel patterned reusable SPR sensor chip}

4-inch glass wafers were purchased from Namkang Hi-Tech co. ltd (Korea). For the fabrication of a reusable SPR chip, the $\mathrm{Cr} / \mathrm{Au}$ film ( $45 \mathrm{~nm} \mathrm{Au}$ on $5 \mathrm{~nm} \mathrm{Cr}$ ) was first prepared on a glass wafer by a thermal evaporation process. Then, $\mathrm{Ni} / \mathrm{Au}$ patterns $(10 \mathrm{~nm} \mathrm{Au}$ on $50 \mathrm{~nm} \mathrm{Ni}$, $5 \mu \mathrm{m} \times 10 \mu \mathrm{m}$ ) were fabricated by the photolithography and thermal evaporation.

\subsection{Conventional SPR sensing using a reusable SPR sensor chip}

The $0.1 \mathrm{mg} / \mathrm{ml}$ solution of protein $\mathrm{G}$ functionalized with cysteine was first flowed for $350 \mathrm{~s}$. Since the cysteine groups formed sulfide or disulfide bonding with a gold surface, the protein $\mathrm{G}$ was attached to the gold surface of a sensor chip (Lee et al., 2007). Afterwards, buffer solution (PBS) was flowed to remove the non-specifically bound protein $\mathrm{G}$ and stabilize a signal. The corresponding C-reactive protein (CRP) antibody solution of $0.1 \mathrm{mg} / \mathrm{ml}$ was flowed for $350 \mathrm{~s}$ to immobilize the antibody molecules on the protein $\mathrm{G}$ molecules. The buffer solution was flowed for $500 \mathrm{~s}$ to remove the non-specifically bound antibody molecules and stabilize a signal. Then, a CRP antigen solution of $5 \mu \mathrm{g} / \mathrm{ml}$ was flowed for $350 \mathrm{~s}$, and the buffer solution was flowed. Here, the signal of CRP antigen is determined by a difference between the signal before and after the passage.

\subsection{Repetitive trap and release of magnetic particles over a Ni patterned} chip

First, the solution of fluorescence labeled magnetic particles was prepared. For trapping the magnetic particles on a Ni patterned chip, the solution was placed on the chip and a magnetic field of $150 \mathrm{mT}$ was applied to the chip for $1 \mathrm{~min}$. Then, it was washed lightly with PBS solution. For releasing the trapped magnetic particles, a magnetic field of $35 \mathrm{mT}$ was applied for $30 \mathrm{~s}$ in the opposite direction to the previous magnetic field, while the chip was washed with DI water.

\subsection{Repetitive sensing process using a reusable SPR sensor chip}

A reusable SPR sensor chip was attached to a conventional SPR chip support by a piece of double-sided adhesive tape. $100 \mu$ l solution of magnetic particles $(0.25 \mathrm{mg} / \mathrm{ml})$ was introduced on the sensor chip, and an external magnetic field of $150 \mathrm{mT}$ was applied for $1 \mathrm{~min}$ by a neodymium magnet. Then, the sensor chip was gently washed with PBS solution. The remaining solution was removed by a science wiper (Yuhan-Kimberly, model Kimtech Science Wiper). The sensor chip was installed on a protective sheath. Then, it was inserted to a conventional SPR detection system (Biacore T100, GE Healthcare). In the system, buffer solution (PBS) flow was first used for a few minutes to stabilize sensor signals. After stabilization of sensor signals, antibodies were immobilized on the magnetic particles following an amine coupling manual (Fischer, 2010). Mixed solution (0.1 M EDC and $0.4 \mathrm{M}$ NHS) was introduced for $5 \mathrm{~min}$ to the sensor chip for activating the carboxyl group of magnetic particles. Then, antibody solution of $50 \mu \mathrm{l} / \mathrm{ml}$ was introduced for $5 \mathrm{~min}$ to the sensor chip. Within $5 \mathrm{~min}$, SPR signals were saturated, indicating that antibody molecules were fully immobilized on the magnetic particles (Fig. S1 in Supplymentary Material). However, it is worth mentioning that some antibody molecules can be adsorbed in a wrong orientation due to the non-selective bindings of linker molecules and they may not function properly, which can be improved by utilizing other more selective linkers such as aptamers or affimers (Centi et al., 2007; Li et al., 2016; Xie et al., 2017). To block the remaining activated carboxyl groups of the magnetic particles, $1 \mathrm{M}$ ethanolamine solution was injected to the sensor chip 
for $1 \mathrm{~min}$. For the detection of target molecules, target molecules were introduced to the sensor chip for $300 \mathrm{~s}$. Then, buffer solution was introduced to the sensor chip for $200 \mathrm{~s}$ to remove target molecules bound non-specifically to the sensor chip. We repeated the detection process with target solution having different concentrations of target molecules. After sensing, we took out the sensor chip from the SPR detection system. Then we applied a magnetic field of $35 \mathrm{mT}$ in an opposite direction to the previous magnetic field. As a result, the used magnetic particles were removed from the chip surface. Then, the sensor chip was washed with DI water for $1 \mathrm{~min}$ and dried. In order to repeat the sensing process, the process described above was repeated.

\section{Results and discussion}

\subsection{Repeated sensing processes of a reusable SPR sensor chip}

Fig. 1 shows a schematic diagram depicting repeated sensing processes of a reusable SPR sensor chip. The detailed fabrication process of a reusable SPR chip is presented in the Materials and methods section. In brief, a metal film ( $45 \mathrm{~nm} \mathrm{Au}$ on $5 \mathrm{~nm} \mathrm{Cr}$ ) was first deposited on a glass substrate. Then, Ni/Au pattern arrays (10 nm Au on $50 \mathrm{~nm}$ $\mathrm{Ni}, 5 \mu \mathrm{m} \times 10 \mu \mathrm{m}$ ) were fabricated on the substrate (Fig. 1A). Here, the Au layer was used to prevent the oxidation of $\mathrm{Ni}$ and to provide stable bindings of biochemical molecules for SPR sensing experiments. The substrate was attached to a sample holder of an SPR measurement system (Biacore, GE Healthcare) using a double-sided adhesive tape (center of Fig. 1). As a first step of sensing, the solution of magnetic particles functionalized with carboxylic groups was first introduced on the chip. Then, an external magnetic field (H-field, $150 \mathrm{mT}$ ) was applied on the substrate with perpendicular direction to the chip (Fig. 1B). Here, the external magnetic field magnetized the ferromagnetic Ni patterns (red arrow) and made magnetic fields near the patterns stronger than other regions. As a result, the magnetic particles were attracted toward the Ni patterns and trapped on them. The chip with trapped magnetic particles was washed with PBS to leave only the magnetic particles bound strongly on the magnetized Ni patterns. Note that the ferromagnetic Ni patterns can hold the trapped magnetic particles even without external magnetic fields. The substrate was inserted into a SPR detection system. In the SPR detection system, PBS solution flowed through a microfluidic channel to stabilize a sensor signal. Then, antibodies were immobilized on the magnetic particles by using EDC and NHS (Fig. 1C). After that, a target molecule solution was introduced for a specific sensing (Fig. 1D). In the sensing process, refractive index changes near the sensor chip surface were measured in a unit of RU (resonance unit). After the sensing, the sensor chip was taken out from the SPR detection system. For the removal of used magnetic particles, an external magnetic field with an opposite direction to the previous applied magnetic field was applied to the sensor chip (Fig. 1E). In this case, the external magnetic field was not strong enough to change the direction of magnetization of the Ni patterns. Thus, total magnetic field above the Ni patterns became weaker than those of other regions. Due to the magnetic field gradient, the magnetic particles were washed out from the sensor chip with DI water. As a result, we could recover a fresh surface of the sensor chip without magnetic particles for the next sensing measurements. In our method, magnetic fields were used to remove the used magnetic particles without any chemical treatment, which reduced chemical contamination on the SPR sensor surface. Furthermore, an external magnetic field doesn't have to be maintained during the SPR sensing process so that our reusable SPR sensor chip can be used in a conventional SPR sensing system.

\subsection{Characterization of a SPR sensor chip with ferromagnetic Ni patterns}

Fig. 2A shows the optical image of a reusable SPR sensor chip. A dark square region in Fig. 2A (i) is a ferromagnetic nickel pattern ar- ray. Fig. 2A (ii) is the magnified image of Ni patterns. White squares are Ni patterns whose size are about $10 \mu \mathrm{m} \times 5 \mu \mathrm{m}$. These patterns were used for the trapping and releasing of magnetic particles. These results indicate that the sizes of $\mathrm{Ni}$ patterns and distances between them are uniformly controlled. The uniformly controlled Ni patterns evenly deflect an applied external magnetic field. As a result, they can hold magnetic particles without a large aggregation (Yoo et al., 2016).

To confirm that the fabricated reusable SPR sensor chip shows similar SPR sensing signals as a conventional SPR sensor chip, we performed a conventional SPR sensing process using a reusable SPR sensor chip (Fig. 2B). The experimental procedure is in the Methods section. The SPR signal image shows increased SPR signals when protein G, C-reactive protein (CRP) antibody, and CRP antigen solutions were introduced on the sensor chip. These results indicate that the measured SPR signals from our reusable SPR sensor chip were similar to measured SPR signals from a conventional SPR sensor chip, even with a Ni pattern array fabricated on our reusable SPR sensor chip.

\subsection{Magnetic trapping and releasing of magnetic particles with a reusable SPR sensor chip}

Fig. 3A shows the simulation result of magnetic field distributions according to the applied magnetic fields at $1 \mathrm{~mm}$ above Ni patterns and the schematic diagrams of a simulation situation. The brighter regions represent those with a stronger magnetic field. Fig. 3A (i) shows a magnetic field distribution when a strong magnetic field was applied to ferromagnetic Ni patterns. The magnetic fields of the regions above the $\mathrm{Ni}$ patterns are stronger than those of the surrounding regions. Since magnetic fields caused by the magnetization of Ni patterns were added to the external magnetic field, the total magnetic fields near the patterns became relatively stronger than those of other regions. This magnetic field gradient generated a magnetic force that attracts paramagnetic materials such as magnetic particles. On the other hand, Fig. 3A (ii) shows a result when a rather weak magnetic field in the opposite direction to the previous applied magnetic field is applied to the Ni patterns. The magnetic field of the region above the Ni pattern is weaker than that of the surrounding portion. Since ferromagnetic materials have hysteresis characteristics for magnetization, they maintain the magnetization direction. Therefore, the magnetic field near the nickel pattern is reduced and becomes weaker than that of the surrounding area. This forces the magnetic particles on the nickel pattern to come out of the pattern. This simulation results show that we can control the trap and release of magnetic particles on ferromagnetic patterns via external magnetic fields (Yoo et al., 2016).

Fig. 3B shows the fluorescence images of repeated trap and release of magnetic particles over a Ni patterned chip. The experimental procedure is in the Methods section. The above trap and release processes were repeated twice. The bright regions represent the trapped magnetic particles on Ni patterns. The above results show that the magnetic particles can be efficiently trapped and released repeatedly.

Fig. 3C shows SEM images taken after trapping magnetic particles on a Ni pattern (i), and releasing them (ii). The trapped magnetic particles are clearly shown in Fig. 3C (i) (marked by red arrows). Fig. 3C (i) shows that the magnetic particles were selectively trapped in the $\mathrm{Ni}$ pattern. Also, the magnetic particles were somewhat evenly distributed on the Ni pattern without large aggregations. As a result, our reusable SPR sensor chip with trapped magnetic particles can have optical properties similar to those of a bare Au film deposited on a glass substrate. It also should be mentioned that the coverage of nanoparticles on a single Ni pattern may not be uniform presumably due to some variations of individual patterns. However, since there are 375,000 of such $\mathrm{Ni}$ patterns on our sensor chip, we could still obtain uniform coverage of nanoparticles over the entire sensor surface (Yoo et al., 2016). On 

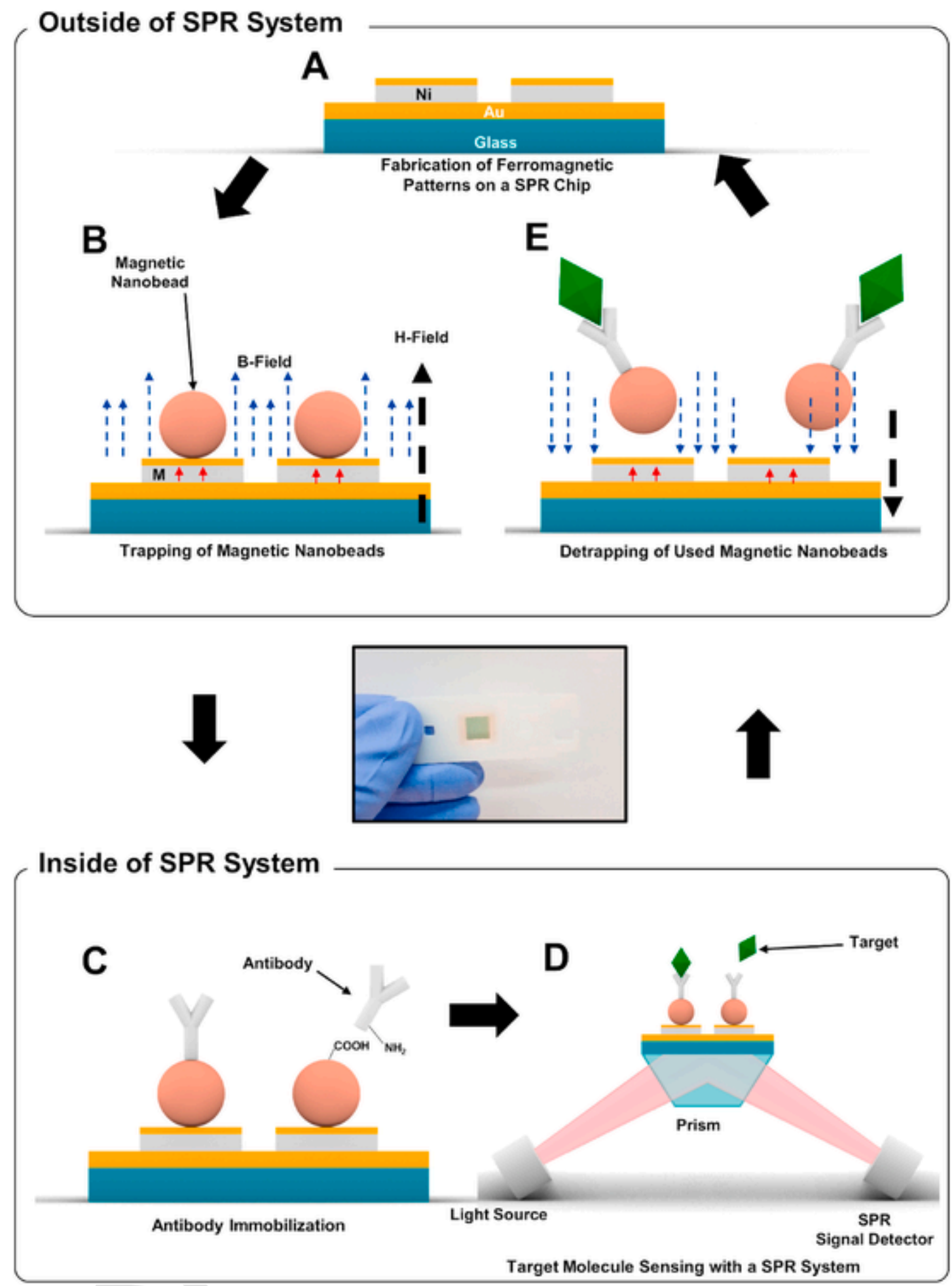

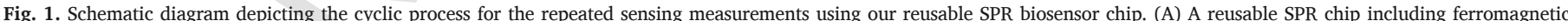

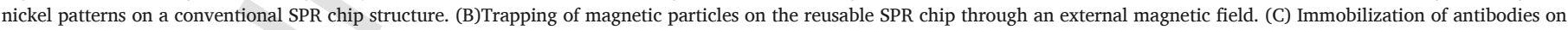

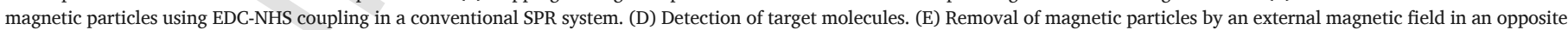
direction to that for trapping.

the other hand, in Fig. 3C (ii) the magnetic particles have clearly removed, indicating that our reusable SPR sensor chip can be reused for additional sensing processes.

Fig. 3D shows UV-VIS transmission spectra of an Au film, a Ni patterned $\mathrm{Au}$ film and a Ni patterned Au film with trapped magnetic particles. All the $\mathrm{Au}$ films were deposited on a glass substrate. Note that the transmittance intensity decreased after Ni patterning and magnetic particle trapping because the deposited $\mathrm{Ni}$ films and the trapped magnetic particles somewhat blocked lights (Srivastava et al., 2012; $\mathrm{Su}$ et al., 2005). However, the peak wavelength of the transmissions remained as $\sim 520 \mathrm{~nm}$, which is similar to the previous results (Axelevitch et al., 2013; Siegel et al., 2011). Also, the shape of the transmit- 
A (i)
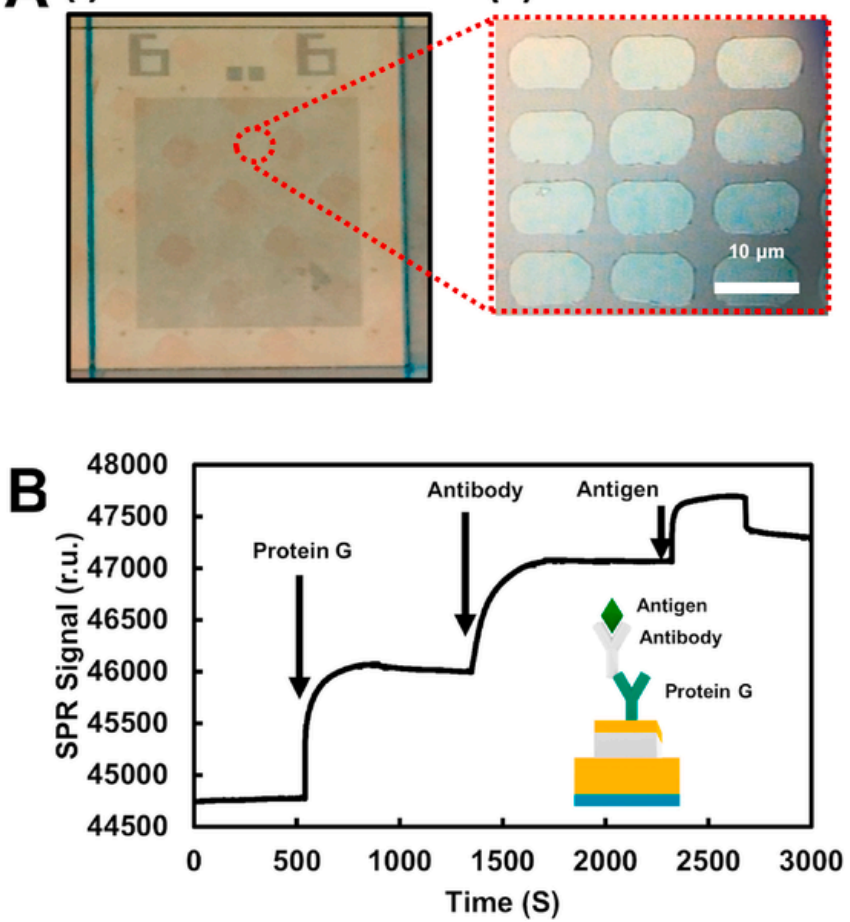

Fig. 2. Characteristics of a reusable SPR sensor chip. (A) Optical image (i) of a reusable SPR sensor chip and magnified optical image (ii) showing Ni patterns. The patterns are uniformly fabricated. (B) Real-time sensing of CRP antigen using a reusable SPR sensor chip in a conventional SPR sensing process. tance curves did not change much even after the deposition of Ni layers and magnetic particles. These results show that the basic optical properties of Au film on a glass substrate were maintained even after Ni patterns were fabricated on Au films or magnetic particles were trapped on the Ni patterns on Au film. In this case, we can expect that SPR signals were not significantly affected because they are based on the change of evanescent waves rather than light transmission (Ekgasit et al., 2005). Therefore, for a sensing process using our reusable SPR sensor chip, we can obtain SPR signals similar to those of a conventional SPR sensing process.

\subsection{Repeated SPR sensing measurements of NP and $I L-13$}

Fig. 4A shows a real-time graph of a sensing process using a reusable SPR sensor chip. Target proteins were nucleoprotein (NP) of H1N1 viruses. The detailed experimental procedure is shown in the Materials and methods section. Here, after the stabilization of the sensor signal, the solution of NP with different concentrations from $300 \mathrm{ng} / \mathrm{ml}$ to $10 \mu \mathrm{g} / \mathrm{ml}$ was sequentially injected to the sensor chip. Note that the SPR signal immediately increases when the NP solution was injected to the sensor chip (Chung et al., 2005; Nanduri et al., 2007). Furthermore, the injection of the NP solution with a larger concentration resulted in a larger increase in the SPR signal. The slight decrease of SPR signals at a high antigen concentration is presumably due to the dissociation of NP and antibodies as reported previously (Scarano et al., 2010). This result shows that our reusable SPR sensor chip could be used to measure the concentration of target molecules in real-time.

Fig. 4B shows SPR sensing results by 7 repeated sensing measurements using a single reusable SPR chip. The detailed experimental procedure is in the Methods section. A $\triangle$ SPR sensing signal was calculated from the difference of SPR signals before NP solution was injected

\section{B}
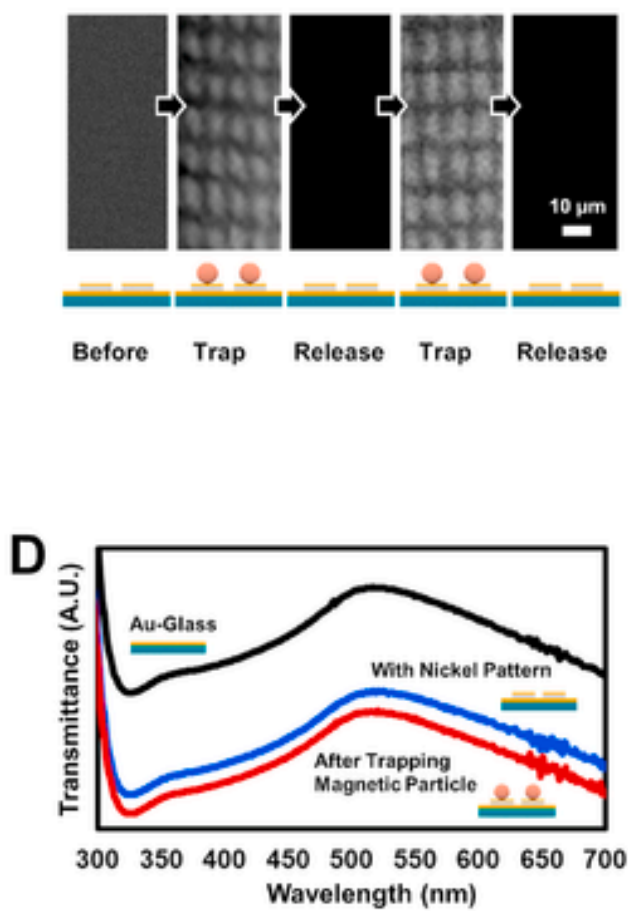

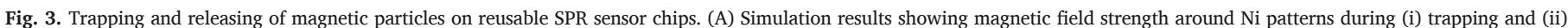

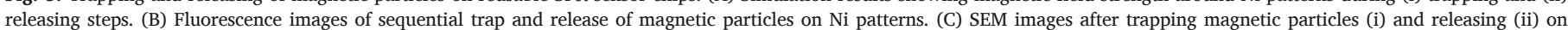

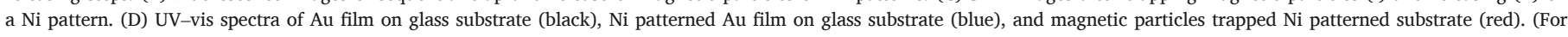
interpretation of the references to colour in this figure legend, the reader is referred to the Web version of this article.) 
to the SPR sensor chip. Note that, as the concentration of Np was increased, $\triangle$ SPR signals increased like a conventional SPR sensor chip (Homola et al., 2002; Nanduri et al., 2007). The average coefficient of variation (CV) was $\sim 12 \%$ until the 5 th repeated sensing measurements, which is in an acceptable range (Jearanaikoon et al., 2016; Wang et al., 2018). In 6th and 7th measurements, the sensor exhibited some fluctuations at a rather low concentration condition with the total average $\mathrm{CV}$ of $\sim 21 \%$, presumably due to some contaminations on the substrate and small signals for low concentration samples. However, at a rather high concentration condition, we could still achieve high accuracy with $9 \%$ of average $\mathrm{CV}$ value for all 7 repeated sensing measurements.

Fig. 4C shows the schematic diagram (i) and the sensing results (ii) of different target molecules using a single reusable sensor chip. Here, we repeated three different sensing measurements using a single SPR chip (Fig. 4C (i)). For the first measurement, the trapped magnetic particles were functionalized with Interleukin 13 (IL-13) antibody and IL-13 antigen solution ranging from $1 \mathrm{pg} / \mathrm{ml}$ to $30 \mathrm{ng} / \mathrm{ml}$ was sequentially measured. In the second measurement, NP solution of $10 \mu \mathrm{g} / \mathrm{ml}$ was measured without any magnetic particle and antibody. For the third measurement, NP solution ranging from $1 \mu \mathrm{g} / \mathrm{ml}$ to $10 \mu \mathrm{g} /$ $\mathrm{ml}$ was sequentially measured with NP antibody. After each measurement was completed, the used magnetic particles were removed via an external magnetic field for an additional sensing measurement. Fig. 4C (ii) shows the normalized $\Delta$ SPR signals from our reusable SPR sensor chip during the repeated measurements of different target molecules with different concentrations. The normalized signals were calculated by normalizing $\Delta$ SPR signals with respect to their maximal $\Delta$ SPR signal values at high concentration conditions. The results of the first measurement show that an increase in concentrations of IL-13 resulted in an increase of a normalized $\triangle \mathrm{SPR}$ signal. By functionalizing the magnetic particles with the IL-13 antibody, we could successfully measure the IL-13 solution with our reusable SPR sensor chip. For the second measurement, the normalized $\triangle \mathrm{SPR}$ signal is negligibly small. This result indicates that the SPR signal increases shown in Fig. 4A was caused by the specific reactions of NP molecules and their antibodies instead of non-specific bindings of NP molecules and Ni patterns. The results of the third measurement are similar to those of the measurements in Fig. 4B. Note that our reusable SPR sensor chip was used to measure two different target molecules (IL-13 in the first measurement and NP in the third measurement), which allowed one to overcome the limitation of a conventional SPR sensor chip. Additionally, although we conducted the first and second measurement before the third measurement, our reusable SPR sensor chip could be used to measure NP solution with reliable results. These results clearly show that our reusable sensor chip can be repeatedly used for sensing various target molecules without the degradation of signals.

\section{Conclusions}

In conclusion, we developed a reusable SPR sensor chip which can be used to repeatedly detect different target molecules in a conventional SPR system. Here, we used ferromagnetic patterns fabricated on a SPR sensor chip to trap a layer of magnetic particles, and those with trapped magnetic particles were used as a substrate for a SPR sensing in a conventional SPR system. Note that the Ni patterns and trapped magnetic particles did not affect the SPR characteristics of our sensor chip. After the sensing was completed, we applied external magnetic fields to the patterns for removing the used magnetic particles. The magnetic particles were completely removed so that we could trap a new layer of fresh magnetic particles on the SPR chip for additional sensing measurements. With only a single reusable SPR sensor chip, we successfully repeated sensing measurements to detect the nucleoprotein (NP) of H1N1 influenza virus for 7 times with high accuracy. With only a single reusable SPR sensor chip, we successfully repeated sensing measurements to detect the nucleoprotein (NP) of H1N1 influenza virus for more than 7 times without significant degradation of sensing signals. Also, we demonstrated that a single SPR chip could be utilized to detect different target molecules. Our method is a powerful strategy which allows one to reuse rather expensive SPR chips in a conventional SPR system and bring them for versatile practical applications such as
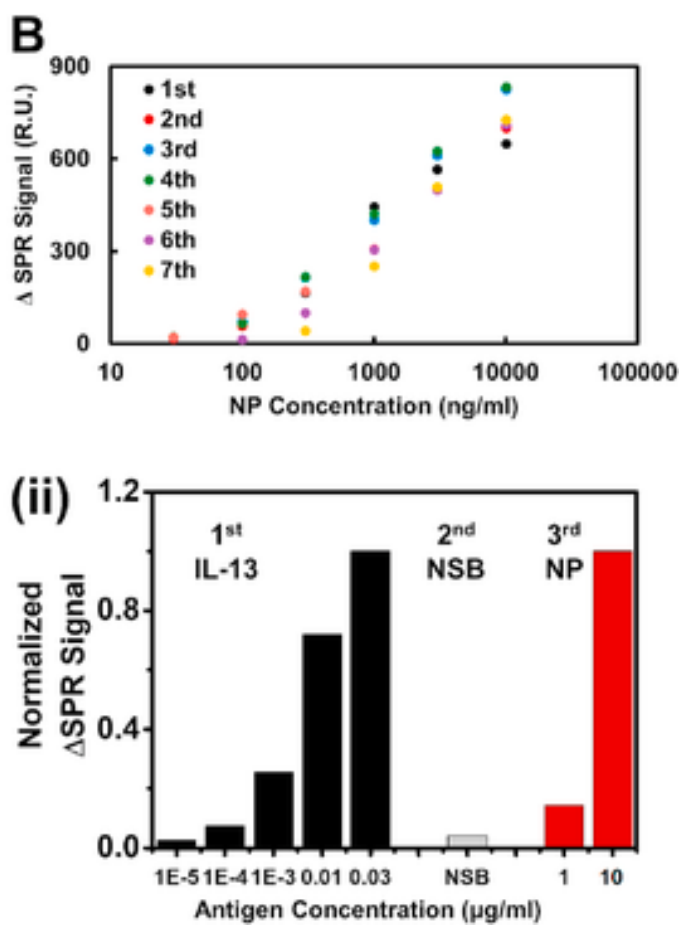

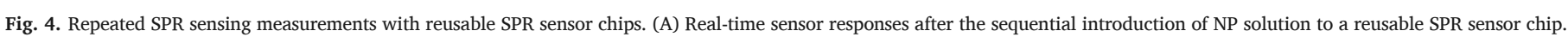

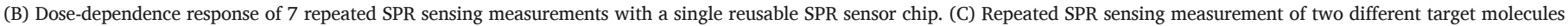
(IL-13, NP) with a single reusable SPR sensor chip. (i) Schematic diagram of the repeated sensing measurement. (ii) Dose-dependence response of the measurement. 
a molecular recognition study, a drug screening, a blood test, and a disease diagnosis.

\section{CRediT authorship contribution statement}

Haneul Yoo: Methodology, Investigation, Writing - original draft, Visualization. Junghyun Shin: Methodology, Investigation, Writing original draft, Visualization. Jieun Sim: Investigation, Visualization. Hyunmin Cho: Investigation, Visualization. Seunghun Hong: Conceptualization, Supervision, Project administration, Funding acquisition.

\section{CRediT authorship contribution statement}

Haneul Yoo: Methodology, Investigation, Writing - original draft, Visualization. Junghyun Shin: Methodology, Investigation, Writing - original draft, Visualization. Jieun Sim: Investigation, Visualization. Hyunmin Cho: Investigation, Visualization. Seunghun Hong: Conceptualization, Supervision, Project administration, Funding acquisition.

\section{Declaration of competing interest}

The authors declare that they have no known competing financial interests or personal relationships that could have appeared to influence the work reported in this paper.

\section{Acknowledgment}

This work was supported by the National Research Foundation of Korea (NRF) funded by the Ministry of Science and ICT (MSIT) of Korea (No. 2013M3A6B2078961 and 2020R1A2B5B02002152), and the European Research Council (ERC) under the European Union's Horizon 2020 research and innovation programme (grant agreement no. 682286). S.H. also acknowledges the support from Samsung Electronics.

\section{Appendix A. Supplementary data}

Supplementary data to this article can be found online at https://doi. org/10.1016/j.bios.2020.112561.

\section{References}

Axelevitch, A, Apter, B, Golan, G, 2013. Simulation and experimental investigation of optical transparency in gold island films. Optic Express 21 (4), 4126-4138.

Centi, S, Tombelli, S, Minunni, M, Mascini, M, 2007. Aptamer-based detection of plasma proteins by an electrochemical assay coupled to magnetic beads. Anal. Chem. 79 (4), 1466-1473.

Chiavaioli, F, Zubiate, P, Del Villar, I, Zamarreno, C R, Giannetti, A, Tombelli, S, Trono, C, Arregui, F J, Matias, I R, Baldini, F, 2018. Femtomolar detection by nanocoated fiber label-free biosensors. ACS Sens. 3 (5), 936-943.

Choi, S, Chae, J, 2009. Reusable biosensors via in situ electrochemical surface regeneration in microfluidic applications. Biosens. Bioelectron. 25 (2), 527-531.

Chung, Ji-won, et al., 2005. Application of SPR biosensor for medical diagnostics of human hepatitis B virus (hHBV). Sensors and Actuators B: Chemical.

Ekgasit, S, Thammacharoen, C, Yu, F, Knoll, W, 2005. Influence of the metal film thickness on the sensitivity of surface plasmon resonance biosensors. Appl. Spectrosc. 59 (5), 661-667.

Fischer, M J E, 2010. Amine coupling through EDC/NHS: a practical approach. In: Mol, N J, Fischer, M J E (Eds.), Surface Plasmon Resonance: Methods and Protocols. Humana Press, Totowa, NJ, pp. 55-73.

Goode, J A, Rushworth, J V H, Millner, P A, 2015. Biosensor regeneration: a review of common Techniques and outcomes. Langmuir 31 (23), 6267-6276.

Guo, T, 2017. Fiber grating-assisted surface plasmon resonance for biochemical and electrochemical sensing. J. Lightwave Technol. 35 (16), 3323-3333.

Homola, J, Dostalek, J, Chen, S F, Rasooly, A, Jiang, S Y, Yee, S S, 2002. Spectral surface plasmon resonance biosensor for detection of staphylococcal enterotoxin B in milk. Int. J. Food Microbiol. 75 (1-2), 61-69.

Hsu, H F, Lin, Y T, Huang, Y T, Lu, M F, Chen, C H, 2015. In situ regeneration of Si-based ARROW-B surface plasmon resonance biosensors. J. Med. Biol. Eng. 35 (3), 305-314.
Jearanaikoon, Patcharee, et al., 2016. The evaluation of loop-mediated isothermal amplification-quartz crystal microbalance (LAMP-QCM) biosensor as a real-time measurement of HPV16 DNA. Journal of Virological Methods.

Jecklin, M C, Schauer, S, Dumelin, C E, Zenobi, R, 2009. Label-free determination of protein-ligand binding constants using mass spectrometry and validation using surface plasmon resonance and isothermal titration calorimetry. J. Mol. Recogn. 22 (4), 319-329.

Karlsson, R, Falt, A, 1997. Experimental design for kinetic analysis of protein-protein interactions with surface plasmon resonance biosensors. J. Immunol. Methods 200 (1-2), 121-133.

Krishnamoorthy, G, Carlen, E T, van den Berg, A, Schasfoort, R B M, 2010. Surface plasmon resonance imaging based multiplex biosensor: integration of biomolecular screening, detection and kinetics estimation. Sensor. Actuator. B Chem. 148 (2), $511-521$.

Lee, J M, Park, H K, Jung, Y, Kim, J K, Jung, S O, Chung, B H, 2007. Direct immobilization of protein $\mathrm{G}$ variants with various numbers of cysteine residues on a gold surface. Anal. Chem. 79 (7), 2680-2687.

Lee, K S, Lee, M, Byun, K M, Lee, I S, 2011. Surface plasmon resonance biosensing based on target-responsive mobility switch of magnetic nanoparticles under magnetic fields. J. Mater. Chem. 21 (13), 5156-5162.

Li, J L, Chang, K W, Wang, C H, Yang, C H, Shiesh, S C, Lee, G B, 2016. On-chip, aptamer-based sandwich assay for detection of glycated hemoglobins via magnetic beads. Biosens. Bioelectron. 79, 887-893.

Loyez, M, Lobry, M, Wattiez, R, Caucheteur, C, 2019. Optical fiber gratings immunoassays. Sens. Basel 19 (11)

Makaraviciute, A, Ramanavicius, A, Ramanaviciene, A, 2015. Development of a reusable protein $\mathrm{G}$ based SPR immunosensor for direct human growth hormone detection in real samples. Anal Methods-Uk 7 (23), 9875-9884.

Mandenius, C F, Wang, R H, Alden, A, Bergstrom, G, Thebault, S, Lutsch, C, Ohlson, S, 2008. Monitoring of influenza virus hemagglutinin in process samples using weak affinity ligands and surface plasmon resonance. Anal. Chim. Acta 623 (1), 66-75.

Nanduri, V, Bhunia, A K, Tu, S I, Paoli, G C, Brewster, J D, 2007. SPR biosensor for the detection of L-monocytogenes using phage-displayed antibody. Biosens. Bioelectron. 23 (2), 248-252.

Patching, S G, 2014. Surface plasmon resonance spectroscopy for characterisation of membrane protein-ligand interactions and its potential for drug discovery. Bba-Biomembr. 1838 (1), 43-55.

Pollet, J, Delport, F, Janssen, K P F, Jans, K, Maes, G, Pfeiffer, H, Wevers, M, Lammertyn, J, 2009. Fiber optic SPR biosensing of DNA hybridization and DNA-protein interactions. Biosens. Bioelectron. 25 (4), 864-869.

Roche, P J R, Cheung, M, Wang, S, Banan, B, Chodavarapu, V, Kirk, A, 2011. Demonstration of a Reusable Plasmonic Polymer Microarray Sensing Platform. SPIE.

Scarano, Simona, et al., 2010. Surface plasmon resonance imaging for affinity-based biosensors. Biosensors and Bioelectronics 25 (5).

Shushama, K N, Rana, M M, Inum, R, Hossain, M B, 2017. Graphene coated fiber optic surface plasmon resonance biosensor for the DNA hybridization detection: simulation analysis. Optic Commun. 383, 186-190.

Siegel, J, Lyutakov, O, Rybka, V, Kolská, Z, Švorčík, V, 2011. Properties of gold nanostructures sputtered on glass. Nanoscale Res. Lett. 6 (1), 96.

Srivastava, M, Singh, J, Yashpal, M, Gupta, D K, Mishra, R K, Tripathi, S, Ojha, A K, 2012. Synthesis of superparamagnetic bare $\mathrm{Fe} 3 \mathrm{O} 4$ nanostructures and core/shell (Fe3O4/ alginate) nanocomposites. Carbohydr. Polym. 89 (3), 821-829.

Su, Y K, Chang, P C, Chen, C H, Chang, S J, Yu, C L, Lee, C T, Lee, H Y, Gong, J, Chen, P C, Wang, C H, 2005. Nitride-based MSM UV photodetectors with photo-chemical annealing Schottky contacts. Solid State Electron. 49 (3), 459-463.

Wang, Weiguo, et al., 2018. Ultrasensitive colorimetric immunoassay for hCG detection based on dual catalysis of Au@Pt core-shell nanoparticle functionalized by horseradish peroxidase. Spectrochimica Acta Part A: Molecular and Biomolecular Spectroscopy.

Wang, X P, Zhan, S Y, Huang, Z H, Hong, X Y, 2013. Review: advances and applications of surface plasmon resonance biosensing instrumentation. Instrum. Sci. Technol. 41 (6), 574-607.

Wegner, G J, Lee, N J, Marriott, G, Corn, R M, 2003. Fabrication of histidine-tagged fusion protein arrays for surface plasmon resonance imaging studies of protein-protein and protein-DNA interactions. Anal. Chem. 75 (18), 4740-4746.

Wong, C L, Olivo, M, 2014. Surface plasmon resonance imaging sensors: a review. Plasmonics 9 (4), 809-824.

Xie, C, Tiede, C, Zhang, X, Wang, C, Li, Z, Xu, X, McPherson, M J, Tomlinson, D C, Xu, W, 2017. Development of an Affimer-antibody combined immunological diagnosis kit for glypican-3. Sci. Rep. 7 (1), 9608.

Yoo, H, Lee, D J, Cho, D G, Park, J, Nam, K W, Cho, Y T, Park, J Y, Chen, X, Hong, S, 2016. Magnetically-refreshable receptor platform structures for reusable nano-biosensor chips. Nanotechnology 27 (4).

Zeng, S W, Baillargeat, D, Ho, H P, Yong, K T, 2014. Nanomaterials enhanced surface plasmon resonance for biological and chemical sensing applications. Chem. Soc. Rev. 43 (10), 3426-3452.

Zhao, Q Y, Duan, R Q, Yuan, J L, Quan, Y, Yang, H, Xi, M R, 2014. A reusable localized surface plasmon resonance biosensor for quantitative detection of serum squamous cell carcinoma antigen in cervical cancer patients based on silver nanoparticles array. Int. J. Nanomed. 9, 1097-1104.

Zhao, Y, Tong, R J, Xia, F, Peng, Y, 2019. Current status of optical fiber biosensor based on surface plasmon resonance. Biosens. Bioelectron. 142. 